\title{
Chronological association of public face mask usage with the progression of coronavirus disease 2019 (COVID-19) pandemic in a medium-sized Texas city
}

\author{
Tyler B. Helton MS*, Andrés E. Guerrero-Criado BS, Xinyi Huang MA, Mark Sigler MD
}

\begin{abstract}
The coronavirus disease 2019 (COVID-19) pandemic has brought many public health issues to the forefront. One area of interest is the use of face masks for the reduction in disease dissemination. This observational study surveys the trend of public mask usage in Amarillo, TX, over two months during the COVID-19 pandemic. We hypothesized that public mask usage would decrease during the data collection period due to "COVID Fatigue" in the region studied. For two hours per week, customers entering a local supermarket were counted and recorded as mask wearing or not mask wearing. The percentage of customers wearing masks over time was determined. The regional COVID-19 incidence rate was analyzed during this mask usage trend. A decrease in mask utilization was confirmed throughout May/June 2020 before policy interventions in July. Mask utilization was highest with a peak of 35.5\% in May before a decrease to a floor of $13.9 \%$ in June. A significant increase in mask use following a state mask mandate and private policy change occurred. The mandate alone was not enough to cause a $100 \%$ compliance rate in the population, and the individual store policy change preceded the most significant increase. A strong negative correlation between mask usage and active coronavirus cases in Amarillo was observed. This study revealed a trend of decreasing compliance with mask utilization over time and suggests that maximum compliance with mask use requires a combination of both public health policy and private business policy implementation.
\end{abstract}

Keywords: Masks, trends, coronavirus, public policy, public health, compliance, epidemiology

\section{INTRODUCTION}

During the COVID-19 pandemic, public policy in the United States regarding the general use of face masks in public varied among regions and is likely influenced by policies set by regional and national

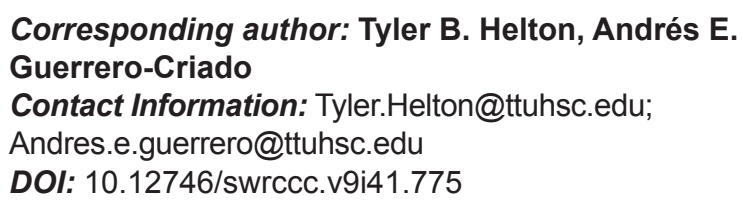

leadership. One factor that significantly influenced face mask policy was the national shortage of Personal Protective Equipment (PPE). ${ }^{1}$ A significant portion of the national PPE stockpile had been exhausted following the $2009 \mathrm{H} 1 \mathrm{~N} 1$ influenza pandemic and had not been replaced before the COVID-19 outbreak. In addition, most PPE products were not produced domestically. ${ }^{2}$ Due to these issues, President Donald Trump invoked the Defense Protection Act (DPA) on March 18, 2020, which gave the president the power

\footnotetext{
${ }^{*}$ All authors contributed equally to this study.
} 
to directly oversee the industrial production of needed products. This Act also served to prevent prices of medical equipment and other necessary goods from inflating during times of emergency. On March 23, 2020, the DPA was activated to direct General Motors to manufacture ventilators. Later, on April 2, 2020, the Act was used again to increase N95 mask and ventilator production. ${ }^{3}$

The Centers for Disease Control and Prevention (CDC) recommended multiple policies to offset the massive PPE shortage. In clinics and hospitals, prolonged use of masks and face shields was recommended. Cloth masks were recommended if no clinical masks were available. Mask decontamination and recycling were employed in many hospitals across the country. ${ }^{4}$ The CDC recommended universal mask wearing in public to reduce disease spread. Despite this, only 12 states in the United States as of May 14, 2020, required universal mask wearing. ${ }^{5}$ According to a national survey done in April 2020 with over 22,000 participants, only $55 \%$ of participants reported following face mask recommendations "very closely." 6 However, an increasing number of states and counties are beginning to require face mask use in public.

Since the onset of the COVID-19 pandemic, a persistent anti-mask sentiment in the United States has been present, and mask use has become controversial. Many citizens decline to comply with recommendations or with local government orders to wear masks in public or to practice social distancing. There have been multiple comments about the use of masks on social media and through daily conversation, and these concerns have led to studies on the efficacy of face mask use by the general public as a preventive measure. One study used laser light scattering to visualize respiratory droplet emission during speech with and without masks. ${ }^{7}$ In this study, a simple cloth mask blocked nearly all of the droplets compared to no mask. This study did not evaluate microdroplets (droplets less than 5 microns) transmission, but current expert opinion maintains that microdroplets are not responsible for the majority of transmission. ${ }^{8}$ Another study by Kumar et al. demonstrated that masks reduce the spread of microdroplets to 1.5 meters (as opposed to
5 meters without a mask), adding support to the efficacy of a simple cloth mask. ${ }^{9}$

In perhaps the most convincing study on face mask efficacy, Leung et al. evaluated live pathogens in 246 patients with viral upper respiratory infections who were randomized into mask and no mask groups. ${ }^{10}$ The groups then collected samples during 30-minute periods. The samples were analyzed for the presence of viral material and demonstrated that surgical masks block $100 \%$ of seasonal coronavirus (NL63, OC43, and HKU1) droplets and aerosols projected during expiration. The research done on this topic shows that the benefit of mask use in preventing COVID disease spread depends on the infected individual's wearing a mask. This benefit does not extend to healthy individuals wearing masks. The question that arises from this information is whether policies should be implemented to encourage or mandate healthy individuals to wear masks.

\section{Methods}

For two hours per week, customers entering a local supermarket were counted and recorded as mask wearing or not mask wearing. The specific times were on Fridays and Sundays from 5:00 to 6:00 p.m. The data collection period was from May 17, 2020, to July 26,2020 . The trend over time was analyzed as a raw data bar graph and as the percentage of customers wearing masks. Customers who had masks pulled down or obviously in their hands were counted as mask wearing and were assumed to be wearing the mask in the store. The regional COVID-19 prevalence was analyzed during this mask use study. Policy changes and any other newsworthy events were also included to identify possible effects on the trend.

\section{RESULTS}

Throughout the data collection period from May 17,2020 to July 26, 2020, 12,383 customers entering a local grocery store were tallied as mask wearing or not mask wearing. Twenty observation sessions of one-hour duration were completed. 


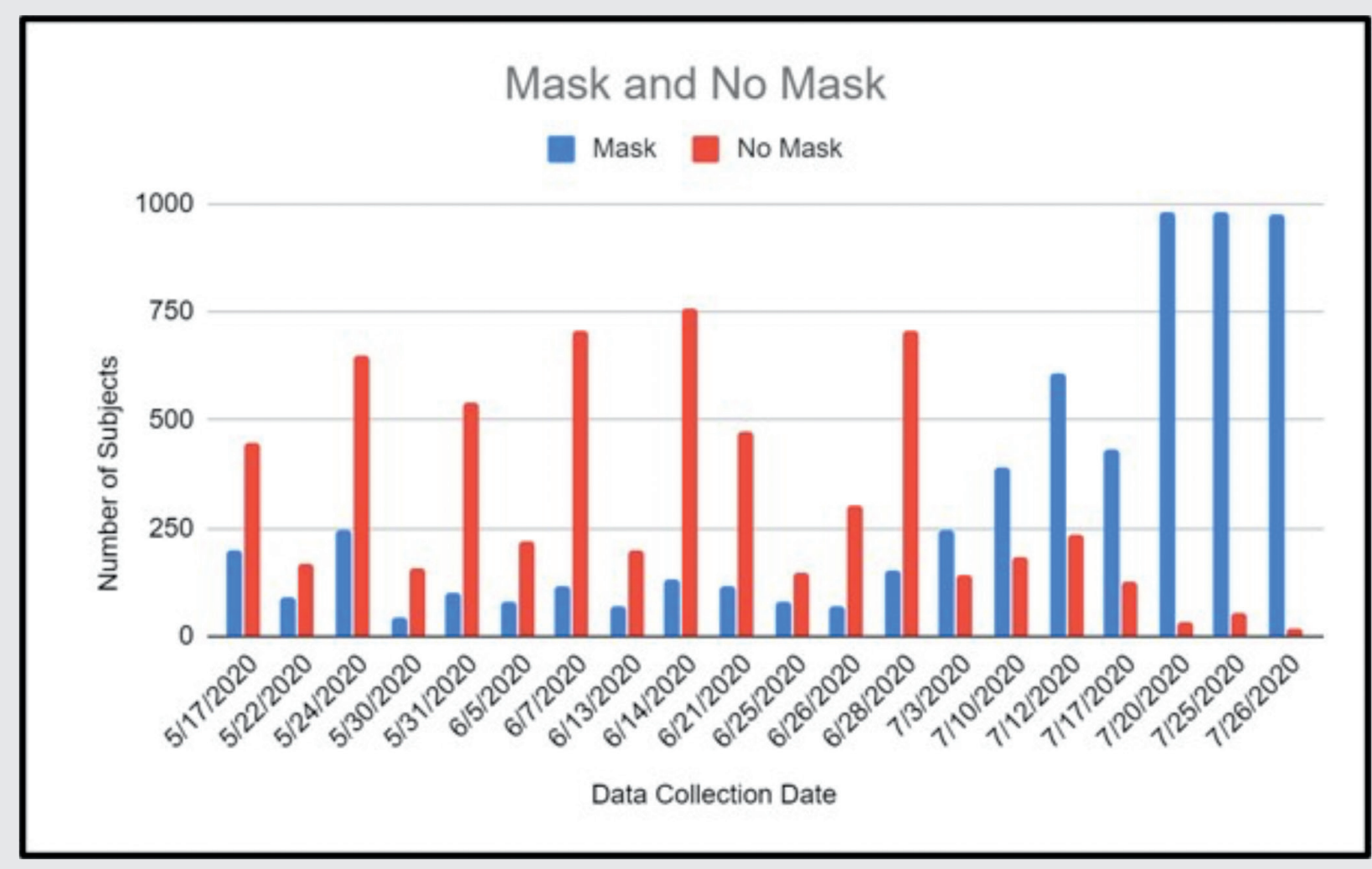

Figure 1. Raw data displayed in bar graph format of the number of customers entering a local Amarillo store wearing masks (blue) versus the number of customers not wearing masks (red). Data collection was from 05/17/2020 to $07 / 26 / 2020$. There were 12,383 customers counted over 20 hours during the data collection period.

In Figure 1, the raw data collected over time are displayed. Data were collected on Fridays and Sundays from 5:00 to 6:00 p.m. Sundays tended to have higher volumes of customers. The times were kept consistent to limit confounders. However, a few days were done at separate times due to weather or other constraints. June 12, 2020, was excluded due to rain and made up on June 13, 2020. June 19, 2020, was excluded due to a missed data collection period and made up on June 25, 2020.

In Figure 2, the number of customers wearing masks is presented as a percentage over time. In late May, mask wearing peaked at $35.5 \%$ but subsequently declined to below $14 \%$ in early June. Throughout the remainder of June, the percentage ranged from $13.9 \%$ (114 mask users out of 818 participants) to $26.1 \%$ (78 mask users out of 299 participants) except for June 25, 2020 , which had an increase to $34.6 \%$ (79 mask users out of 228 participants). June 25th was the day before Texas Governor Greg Abbott issued an executive order to close bars and limit restaurants to $50 \%$ capacity. ${ }^{12}$

Beginning in July, however, the percentage of customers wearing masks significantly increased. This was directly after Texas Governor Greg Abbott issued a statewide mask order on July 2, 2020, suggesting that the mask mandate had a significant effect on the trend. However, the percentage of those wearing masks increased only to $63.6 \%$ (248 mask users out of 390 participants) on the first day after the mandate and to $77.5 \%$ (433 mask users out of 559 participants) in the subsequent weeks. The grocery store being observed issued its policy requiring face masks 


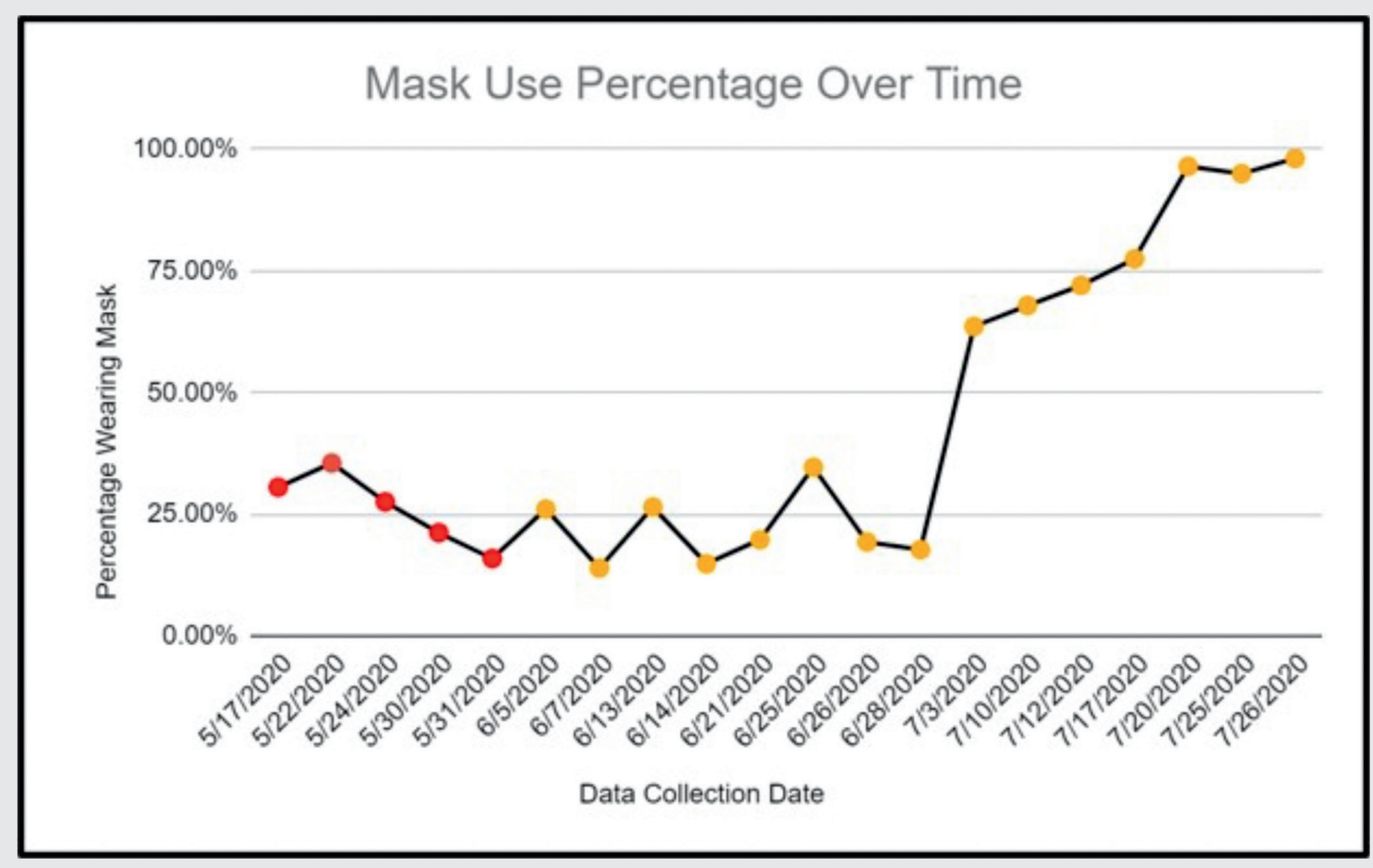

Figure 2. Percentage of customers wearing masks at a local Amarillo grocery store over time. Red dots connote Amarillo area COVID-19 status at "Level Red" while Orange dots connote "Level Orange." Status levels are further detailed by the Amarillo Public Health department. ${ }^{11}$ Data collection was from May 17, 2020, to July 26, 2020. There were 12,283 customers counted over 20 hours during the data collection period.

on July 20, 2020. Following this date, mask usage significantly increased again to $96.5 \%$ (981 mask users out of 1,017 participants) and then to $98.1 \%$ (977 mask users out of 996 participants) on the final day of data collection.

The final factor analyzed was whether there was any correlation between mask use percentage and the number of active cases over time (Figure 3 ). This was done by calculating a Pearson correlation coefficient between the mask use percentage and active coronavirus cases (Figure 4). Data from the Amarillo Public Health Department was compared with the corresponding date from the observation data. Sunday observational data was compared to the following day due to data not being released on the weekend. The result was a Pearson coefficient of -0.76 suggesting a strong negative correlation between mask use and active coronavirus cases.

\section{Discussion}

Amarillo is in the Texas Panhandle, located at latitude $35 \mathrm{~N}$ and longitude $101 \mathrm{~W}$. Interstate 40 runs through the city. Major economic activities include agriculture, petroleum, and meat packing. The estimated population in 2019 was 199,371.

A decrease in mask wearing percentage was detected during the data collection period before Texas Governor Greg Abbott's executive order. It 


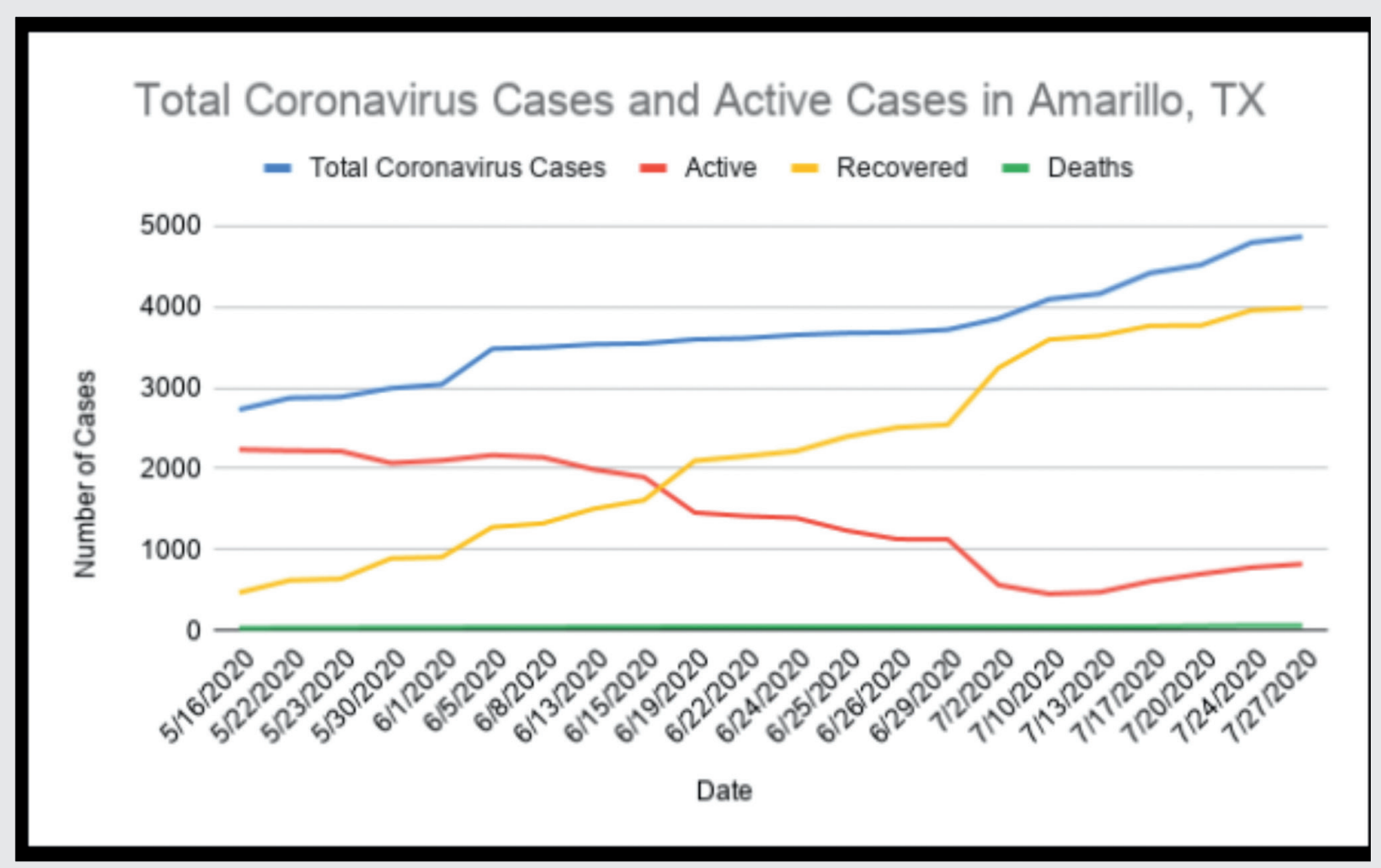

Figure 3. Data published from the Amarillo Public Health Department showing a breakdown of all the known coronavirus cases in Amarillo, TX during the data collection period. ${ }^{13}$

would have been interesting to have an extended data collection period to have detected the trend during the stay at home order from late March to late April. Our survey was able to capture the end of the level red warning and carried throughout the level orange warning in Amarillo. Perhaps the most interesting trend captured was the effect that the statewide mask executive order had on the population followed by the individual policy change of the grocery store. The government policy had a partial effect on compliance whereas the private policy change had near total compliance. An important finding was that the individual policy change did not appear to significantly decrease store traffic. This is of interest to store owners concerned with the economic impact of implementing a mask policy. However, this effect may not be generalized to businesses other than grocery stores.
The strong negative correlation found between mask usage and active coronavirus cases suggests that the increase in mask use may have had a protective effect on disease spread in the Amarillo community. The downward slope of the active cases in Figure 3 coincides with the increase in mask use in Figure 2, particularly the sharp decrease in active cases on July 2, 2020, when Governor Abbott issued the mask order. Many variables could have affected this trend, however, and it is difficult to determine how much the increase in mask use directly impacted the number of active cases. In addition, the number of active cases in this region Texas seems to differ from the number of active cases throughout the state of Texas. ${ }^{14}$

Our study design used prevalence as a metric rather than incidence for several reasons. While incidence, or new cases, would have been preferable to 
Percent Mask Use and Active Cases

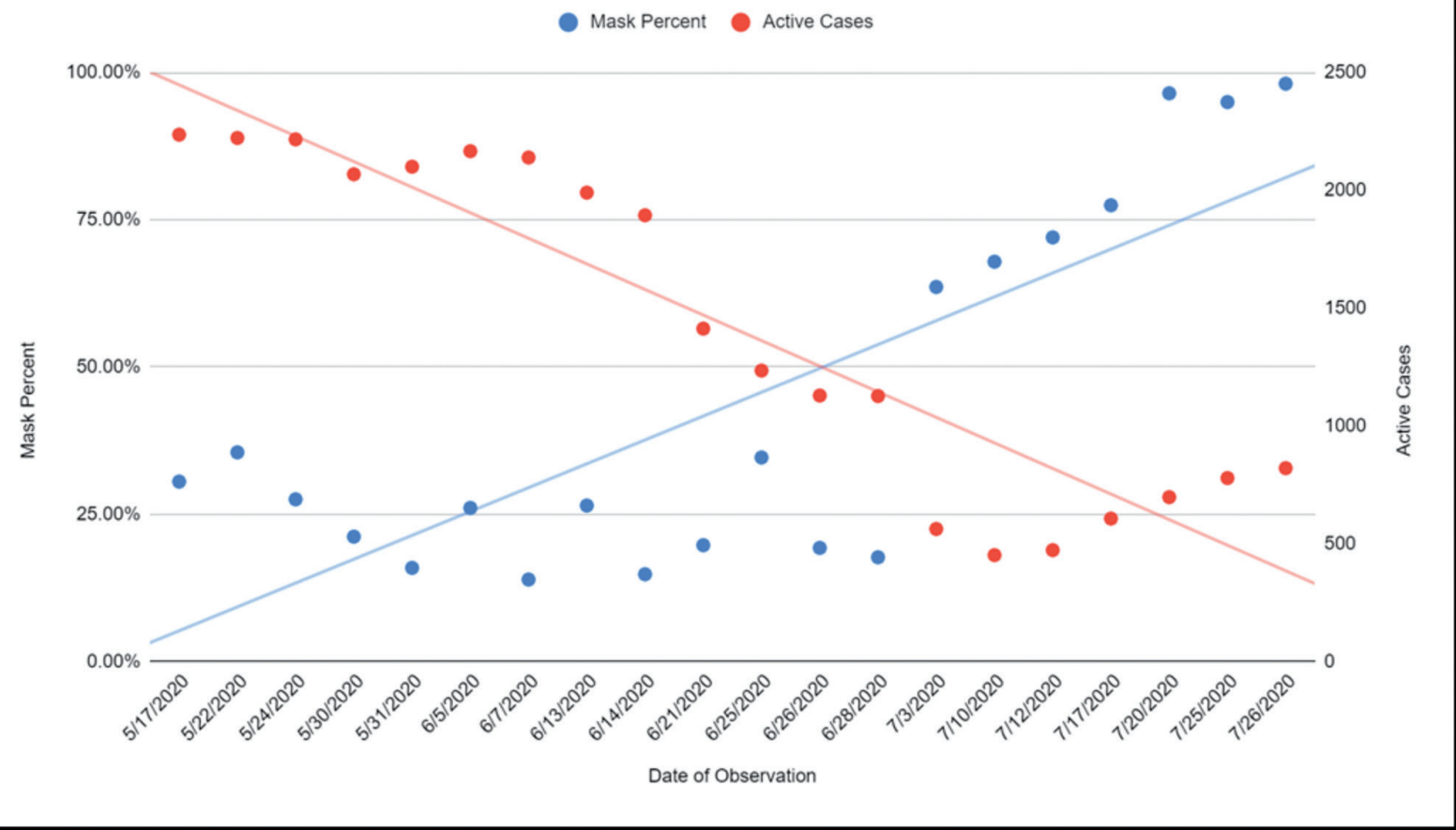

Figure 4. Scatterplot of percent mask use on the left axis and active cases on the right axis over the data collection period. The superimposed trend lines have a negative correlation.

use as a metric, there were a number of limitations and confounders that prevented this. First, the city of Amarillo did not report new cases over the weekend which could introduce skewing of the data if daily new cases were used as a metric. Another factor was that the day of the week could introduce another confounding variable as patients may be more or less likely to present to a clinic depending on the day. Therefore, it was decided to use prevalence as a metric as it serves as an average and would effectively reflect the correlation between mask wearing and disease prevalence. The reasoning was that mask use could decrease the number of new cases while the same number of existing cases are expiring. The decreased number of new cases adding to the active case reservoir would be immediately reflected as a result of increased mask usage. Incidence would have been a preferable metric to use. Due to the limitations of the study, however, prevalence served as an effective substitute.

One limitation of our study was that it involved only one grocery store in Amarillo. This could introduce a socioeconomic bias and make the data less generalizable to the entire population of Amarillo. Multiple grocery stores in the area could have been surveyed to reduce this bias. Another limitation was that customers entering the store without a mask could have applied a mask after entry. The data collection method could not account for this. Individual surveys administered to customers could have corrected this but would have been much more labor intensive and could have introduced a response bias. The availability of testing to the general public was another potential confounder on the data. As more tests became available, more active cases were 
found and could explain some of the rise in active cases at the end of the study. This effect may have been greater than the correlation we found. Inferring general trends from a sampling of measurements was another limitation of this study. The sampling nature of our methodology depends on the assumption that the observed trends are representative of the continuous trends. Another limitation was the inability to correlate total volume of non-mask wearers with the incidence of COVID cases due to the sampling methodology. The use of percentage was used due to this limitation.

\section{Conclusion}

This study provides an estimate of the mask use behavior in Amarillo during the COVID-19 pandemic. Several trends were captured during the data collection period that can help public health officials and researchers when considering policies during pandemics and outbreaks. The hypothesis of capturing a decrease in mask use due to mask fatigue was confirmed throughout May and June before policy interventions in July. Mask use was highest with a peak of $35 \%$ (92 mask users out of 259 participants) in May before a decrease to a floor of $13.9 \%$ (114 mask users out of 818 participants) in June. A significant increase in mask usage following the mask executive order and private store policy change was captured. The executive order alone was not enough to cause a $100 \%$ compliance rate in the population, and the individual store policy change was what caused the most significant increase. A strong negative correlation between mask use and active coronavirus cases in Amarillo suggests a possible protective effect and a decrease in disease spread in this county. This correlation supports the use of masks by the general public during this pandemic.

\section{ACKNOWLEDgements}

We would like to thank the Amarillo Public Health Department for providing the data for Amarillo coronavirus cases and all its diligent work with informing the public.
Article citation: Helton TB, Guerrero-Criado AE, Huang X, Sigler M. Chronological association of public face mask usage with the progression of coronavirus disease 2019 (COVID-19) pandemic in a medium-sized Texas city. The Southwest Respiratory and Critical Care Chronicles 2021;9(41):20-27

From: Department of Internal Medicine (TBH, AE G-C, MS), Texas Tech University Health Sciences Center, Amarillo, Texas; The University of South Carolina $(\mathrm{XH})$, Columbia, South Carolina

Submitted: $10 / 14 / 2020$

Accepted: $10 / 9 / 2021$

Reviewer: Gilbert Berdine MD

Conflicts of interest: none

This work is licensed under a Creative Commons

Attribution-ShareAlike 4.0 International License.

\section{REFERENCES}

1. Board on Health Sciences Policy, Health and Medicine Division, National Academies of Sciences, Engineering, and Medicine. The Nation's Medical Countermeasure Stockpile: Opportunities to Improve the Efficiency, Effectiveness, and Sustainability of the CDC Strategic National Stockpile: Workshop Summary. Washington (DC): National Academies Press (US); 2016.

2. Dai T, Bai G, Anderson GF. PPE supply chain needs data transparency and stress testing. J Gen Intern Med 2020; 35(9):2748-9.

3. Ward A. Coronavirus: The Defense Production Act, explained. Vox. 2020 (https://www.vox.com/2020/3/18/21185333/ coronavirus-defense-production-act-trump)

4. CDC. Strategies for Optimizing the Supply of Facemasks. 2020 (www.cdc.gov/coronavirus/2019-ncov/hcp/ppe-strategy/ face-masks.html)

5. Howard J. Masks help stop the spread of coronavirus the science is simple and I'm one of 100 experts urging governors to require public mask-wearing. 2020 (http:// theconversation.com/masks-help-stop-the-spread-of-coronavirus-the-science-is-simple-and-im-one-of-100-expertsurging-governors-to-require-public-mask-wearing-138507)

6. Lazer D, Baum M, Ognyanova $\mathrm{K}$, et al. The state of the nation: a 50-state covid-19 survey Report \#1. 2020 (http:// dx.doi.org/10.31219/osf.io/arwh3)

7. Anfinrud P, Stadnytskyi V, Bax CE, et al. Visualizing speech-generated oral fluid droplets with laser light scattering. N Engl J Med 2020; 382(21):2061-3. 
8. Evidence shows COVID-19 is almost exclusively spread by droplets.2020(https://www.thestar.com/opinion/letters_to_ the_editors/2020/05/09/evidence-shows-covid-19-is-almostexclusively-spread-by-droplets.html)

9. Kumar V, Nallamothu S, Shrivastava S, et al. On the utility of cloth facemasks for controlling ejecta during respiratory events. 2020 (http://arxiv.org/abs/2005.03444)

10. Leung NHL, Chu DKW, Shiu EYC, et al. Respiratory virus shedding in exhaled breath and efficacy of face masks. Nat Med 2020;26(5):676-80.

11. Amarillo News. COVID-19 Status Chart Announced. City of Amarillo, TX 2020 March 16 (www.amarillo.gov/Home/ Components/News/News/864/16)
12. Neilson S, Woodward A, Mosher D. A comprehensive timeline of the coronavirus pandemic at 9 months, from China's first case to the present. Business Insider. 2020 (https://www. businessinsider.com/coronavirus-pandemic-timeline-historymajor-events-2020-3)

13. Amarillo News. COA Covid-19 Report Card. City of Amarillo, TX 2020 July 27 (www.amarillo.gov/Home/Components/ News/News/966/16)

14. Berdine G. Delta variant is in decline in the US, Texas, and Lubbock County. The Southwest Respiratory and Critical Care Chronicles 2021;9 (41) (this issue). 